\title{
Small Triangle-Free Configurations of Points and Lines*
}

\author{
Marko Boben, ${ }^{1}$ Branko Grünbaum, ${ }^{2}$ Tomaž Pisanski, ${ }^{1}$ and Arjana Žitnik ${ }^{1}$ \\ ${ }^{1}$ Faculty of Mathematics and Physics, University of Ljubljana, \\ 1000 Ljubljana, Slovenia \\ \{Marko.Boben,Tomaz.Pisanski,Arjana.Zitnik\}@fmf.uni-lj.si \\ ${ }^{2}$ Department of Mathematics, University of Washington, \\ Seattle, WA 98195, USA \\ grunbaum@math.washington.edu
}

\begin{abstract}
In the paper we show that all combinatorial triangle-free configurations for $v \leq 18$ are geometrically realizable. We also show that there is a unique smallest astral $\left(18_{3}\right)$ triangle-free configuration and its Levi graph is the generalized Petersen graph $G(18,5)$. In addition, we present geometric realizations of the unique flag transitive triangle-free configuration $\left(20_{3}\right)$ and the unique point transitive triangle-free configuration $\left(21_{3}\right)$.
\end{abstract}

\section{Introduction}

Close to a century ago, Steinitz [25, p. 490] stated that it appears that all combinatorial configurations $\left(v_{3}\right)$ with $v>10$ can be realized by geometric configurations of points and straight lines, although this question has not been decided so far. Steinitz's expectation was contradicted a few years ago by an example with $v=16$ (see [8], as well as [2]), which can be easily modified to yield examples for all $v \geq 16$. However, a modified version of this question, which is formulated below after the necessary definitions are presented, is still open.

There exist methods (see, for example, [4] and [26]) and algorithms (such as VEGA, see [17] and [19]) that can be used to decide whether a given combinatorial configuration can be realized geometrically. Since the number of combinatorial configurations grows very fast with $v$ (see below, Table 1, adapted from [1]), it is not feasible to check the realizability of all configurations $\left(v_{3}\right)$ with a given reasonably large $v$. It is therefore of

* The third author was supported in part by a research grant from Ministrstvo za šolstvo, znanost in šport Republike Slovenije. Part of the research was conducted while he was a Neil R. Grabois Visiting Professor of Mathematics at Colgate University. 
Table 1. The numbers of various combinatorial $\left(v_{3}\right)$ configurations (from [1]; the entries for $v=19$ in columns (a) and (b) were obtained later by the same computer program).

\begin{tabular}{rrrrr}
\hline$v$ & (a) & (b) & (c) & (d) \\
\hline 7 & 1 & 1 & 0 & 0 \\
8 & 1 & 1 & 0 & 0 \\
9 & 3 & 3 & 0 & 0 \\
10 & 10 & 10 & 0 & 0 \\
11 & 31 & 25 & 0 & 0 \\
12 & 229 & 95 & 0 & 0 \\
13 & 2,036 & 365 & 0 & 0 \\
14 & 21,399 & 1,432 & 0 & 0 \\
15 & 245,342 & 5,799 & 1 & 1 \\
16 & $3,004,881$ & 24,092 & 0 & 0 \\
17 & $38,904,499$ & 102,413 & 1 & 0 \\
18 & $530,452,205$ & 445,363 & 4 & 0 \\
19 & $7,640,941,062$ & $1,991,320$ & 14 & 0 \\
20 & $?$ & $?$ & 162 & 1 \\
21 & $?$ & $?$ & 4,713 & 1 \\
\hline
\end{tabular}

Column (a) contains the numbers of combinatorial $\left(v_{3}\right)$ configurations, column (b) the numbers of self-polar $\left(v_{3}\right)$ configurations, column (c) the numbers of triangle-free configurations, and column (d) the numbers of triangle-free "point" transitive configurations.

some interest to investigate the realizability of certain special configurations, endowed with unusual properties. One of the reasons is that very frequently configurations (or other mathematical entities) that are special in some respect are also special in other ways as well.

We chose to investigate combinatorial configurations $\left(v_{3}\right)$ which are exceptional in that they contain no triangles. (A triangle in a configuration is a triplet of points, not on one line of the configuration, such that each pair does lie on a line of the configuration.) Relying on data from [1], we investigate the triangle-free combinatorial configurations $\left(v_{3}\right)$ with $v \leq 18$ on their realizability as geometric configurations, and, in particular, on the possibilities of finding symmetric realizations. The same applies to the triangle-free configuration $\left(20_{3}\right)$ that is flag transitive and to the triangle-free configuration $\left(21_{3}\right)$ that is point transitive; by the data in Table 1 these two configurations are unique. As we shall see, the expectations of finding interesting properties is fulfilled.

The paper is organized as follows. Section 2 describes the combinatorial prerequisites, Section 3 the geometric ones. Section 4 presents the results of a detailed examination of the eight configurations under consideration. The last two sections give additional data and historical information, as well as some open problems.

\section{Combinatorial Configurations}

In this paper by combinatorial configuration or more specifically combinatorial $\left(v_{3}\right)$ configuration $\mathcal{C}$ we mean an incidence structure of $v$ symbols called "points" and $v$ 
symbols called "lines", such that three "lines" are incident with each "point", three "points" with each "line", and two "lines" meet in at most one "point". No geometric significance is attached to the terms "point" and "line". It is often convenient to identify the "lines" of a combinatorial $\left(v_{3}\right)$ configuration with the triplet of "points" incident with it. When there is no danger of confusion, we dispense with the quotation marks.

The known numbers of different (that is, nonisomorphic) combinatorial $\left(v_{3}\right)$ configurations are shown in Table 1, together with the available information about the triangle-free ones among them. While the entries for $v \leq 11$ were determined already in the nineteenth century, the other entries are very recent. ([10] for $v \leq 14$, [1] for the other values). The numbers in the last two columns show how special the triangle-free configurations are.

Combinatorial configurations are closely related to graphs. A Levi graph $L(\mathcal{C})$ (or incidence graph) of a $\left(v_{3}\right)$ configuration $\mathcal{C}$ (or of any incidence structure with points and lines) is a bipartite graph with black vertices representing the points, white vertices representing the lines, and with an edge joining two vertices if and only if the corresponding point and line are incident. Combinatorial configuration can be characterized by their Levi graphs in the following way.

Proposition 1. An incidence structure $\mathcal{C}$ is a $\left(v_{3}\right)$ configuration if and only if its Levi graph is trivalent and has girth (the length of the shortest cycle) at least 6.

For the proof of the proposition and more about the relation between configurations and graphs, see, for example, [5]. The term Levi graph was introduced in this paper; the concept seems to have originated in the little-known work [15] by Levi who was also the author of the first book on configurations [14].

A cycle of length $2 d$ in the Levi graph $L(\mathcal{C})$ corresponds to a $d$-gon in the combinatorial configuration $\mathcal{C}$. In particular, a hexagon in $L(\mathcal{C})$ corresponds to a triangle in $\mathcal{C}$. A configuration $\mathcal{C}$ is triangle-free if and only if its Levi graph has girth at least 8 .

The primary purpose of this paper is to provide data on the triangle-free combinatorial configurations $\left(v_{3}\right)$ with $v \leq 18$. These previously unpublished data were obtained by [1] in the course of calculations for the tables in that paper. They are presented in Section 4 , in a somewhat modified form, together with the data on the configurations $\left(20_{3}\right)$ and $\left(21_{3}\right)$ which we consider.

A configuration $\mathcal{C}$ is said to be $k$-connected if its Levi graph $L(\mathcal{C})$ is $k$-connected. This is equivalent to saying that the deletion of any fewer than $k$ elements (points and/or lines) from $\mathcal{C}$ does not disconnect $\mathcal{C}$. All combinatorial $\left(v_{3}\right)$ configurations with $v \leq 13$ are 3 -connected, and so are most of the configurations enumerated in Table 1. In particular, all the triangle-free configurations listed in Section 4 are 3-connected.

The generalized Petersen graph $G(n, r)$, introduced by Watkins [28] is a graph with vertex set

$$
V(G(n, r))=\left\{u_{0}, u_{1}, \ldots, u_{n-1}, v_{0}, v_{1}, \ldots, v_{n-1}\right\}
$$

and edge set

$$
E(G(n, r))=\left\{u_{i} u_{i+1}, u_{i} v_{i}, v_{i} v_{i+r}: i=0, \ldots, n-1\right\}
$$


Since $G(n, r)$ is isomorphic to $G(n, n-r)$ and since $G(n, n / 2)$ is not a simple graph, without loss of generality we assume that $0<r<n / 2$. The graphs $G(n, r)$ constitute a standard family of graphs which is a generalization of the renowned Petersen graph $G(5,2)$.

It is well known, see [9], that the generalized Petersen graph $G(n, r)$ is:

(i) vertex transitive if and only if either $n=10$ and $r=2$, or $r^{2} \equiv \pm 1 \quad(\bmod n)$;

(ii) a Cayley graph if and only if $r^{2} \equiv 1(\bmod n)$;

(iii) flag-transitive only in the following seven cases: $(n, r)=(4,1)$-the cube, $(5,2)$ - the Petersen graph, $(8,3)$ - the Moebius-Kantor graph, $(10,2)$ - the dodecahedron, $(10,3)$ - the Desargues graph, $(12,5)$ - the Levi graph of one of the self-polar $\left(12_{3}\right)$ configurations, and $(24,5)$.

For $(24,5)$, see in particular [6].

The family of generalized Petersen graphs $G(n, r)$ contains some other very important graphs, such as the $n$-prism $G(n, 1)$, the Dürer graph $G(6,2)$, etc. We shall show that the graph $G(18,5)$ also deserves to be considered as special.

\section{Geometric Configurations}

A geometric $\left(v_{3}\right)$ configuration is a set of $v$ points and $v$ (straight) lines in the Euclidean plane, such that precisely three of the lines pass through each of the points, and precisely three of the points lie on each of the lines. It is clear that each geometric configuration determines a combinatorial configuration, while the reverse is not true. For example, it is well known that the combinatorial Fano configuration, the only combinatorial $\left(7_{3}\right)$ configuration (projective plane of order 2), cannot be realized (with points and lines) in the Euclidean plane. It is also known that the unique combinatorial $\left(8_{3}\right)$ configuration is not realizable. However, with the exception of a single $\left(10_{3}\right)$ configuration, all 3-connected $\left(v_{3}\right)$ with $v \geq 9$ for which the realizability has been decided, can be geometrically realized in the plane. Indeed, we make the following conjecture.

Conjecture 1. All 3-connected $\left(v_{3}\right)$ configurations with $v>10$ are geometrically realizable by points and straight lines.

We note that Sturmfels and White [26] showed the validity of this conjecture for $v=11$ and 12. In fact, they established the much stronger result that all such configurations can be realized by points and lines in the rational plane. Probably all configurations mentioned in the conjecture can be realized even in the rational plane.

As a small step towards the verification of our conjecture we shall show that all six triangle-free $\left(v_{3}\right)$ configurations with $v \leq 18$ are realizable as are the unique $\left(20_{3}\right)$ and $\left(21_{3}\right)$ considered here.

We note that geometric realizability of a $\left(v_{3}\right)$ configuration in the (real or rational) Euclidean plane is equivalent to its realizability in the corresponding projective plane. We work exclusively in the real Euclidean plane, since this allows us to look for geometric realizations with isometric symmetries. Note that with the term symmetry, we 
refer exclusively to isometric symmetries of geometric configurations, while we use automorphism when we talk about combinatorial configurations.

Symmetries of a geometric configuration are reflected in the automorphisms of the corresponding combinatorial configuration. Loss of symmetry may occur when passing from a combinatorial to a geometric configuration-if the latter exists at all.

Symmetries make the configurations not only more appealing visually, but also help in drawing them and understanding their structure. In particular, we are interested in astral configurations, introduced in [11].

A $\left(v_{3}\right)$ configuration is said to be astral provided its points form two orbits under its groups of (isometric) symmetries, and the lines form two orbits as well. Clearly, no $\left(v_{3}\right)$ configuration can have a single orbit of points or of lines under its symmetries. If both the points and the lines of a $\left(v_{3}\right)$ configuration form at most three orbits, the configuration is called stellar.

\section{Small Triangle-Free Combinatorial $\left(v_{3}\right)$ Configurations}

We come now to the main topic of our paper, the triangle-free configurations with relatively few points. There are six such configurations-one $\left(15_{3}\right)$ configuration, one $\left(17_{3}\right)$ configuration, and four $\left(18_{3}\right)$ configurations-which we consider in turn. We also consider the unique flag transitive triangle-free $\left(20_{3}\right)$ configuration and the unique point transitive triangle-free $\left(21_{3}\right)$ configuration. We find that each of them has some interesting features. Since the triangle-free $\left(15_{3}\right)$ configuration and the triangle-free $\left(17_{3}\right)$ configuration are the only combinatorial triangle-free configurations with the given number of points, and since the property of being triangle-free is (combinatorially and geometrically) self-dual, these configurations are necessarily combinatorially self-polar. Considering the four triangle-free $\left(18_{3}\right)$ configurations, two of them are self-polar. All eight configurations can be realized geometrically, as shown in Figs. 2, 4, 6, 8, 10, 12, 14, 15, and 17.

\subsection{The Triangle-Free Configuration $\left(15_{3}\right)$}

The self-polar triangle-free configuration $\left(15_{3}\right)$ is often called the Cremona-Richmond configuration. It is highly symmetric: its automorphism group is $S_{6}$, the symmetric group of degree 6, and is transitive on its flags. Its Levi graph (see Fig. 1) is the remarkable and well-known Tutte 8-cage. There is a one-parameter family of different stellar realizations. One stellar realization is shown in Fig. 2. It can be proved that there is no astral realization of this configuration. The Cremona-Richmond configuration $\left(15_{3}\right)$ has lines:

$\begin{array}{ccccccccccccccc}1 & 2 & 3 & 5 & 4 & 7 & 6 & 11 & 10 & 9 & 8 & 15 & 14 & 13 & 12 \\ 1 & 1 & 1 & 2 & 2 & 3 & 3 & 4 & 4 & 5 & 5 & 6 & 6 & 7 & 7 \\ 2 & 4 & 6 & 8 & 10 & 12 & 14 & 8 & 10 & 9 & 11 & 8 & 10 & 9 & 11 \\ 3 & 5 & 7 & 9 & 11 & 13 & 15 & 12 & 14 & 13 & 15 & 15 & 13 & 14 & 12\end{array}$

It is flag transitive and combinatorially self-polar (that is, has a self-duality of order 2). This self-polarity is given by the correspondence $1 \leftrightarrow 1,2 \leftrightarrow 2,3 \leftrightarrow 3, \ldots$ None 


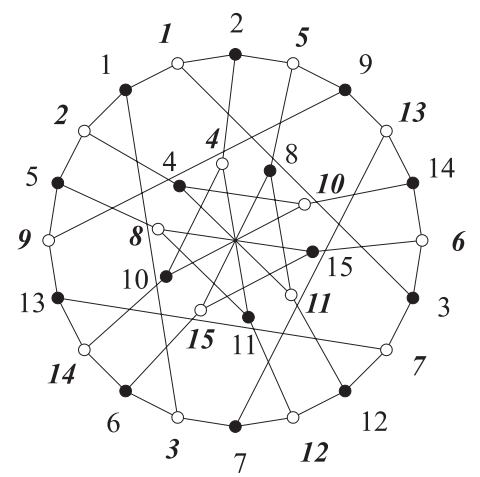

Fig. 1. The Levi graph of the triangle-free combinatorially self-polar Cremona-Richmond $\left(15_{3}\right)$ configuration. This graph is the Tutte 8-cage.

of the other $\left(v_{3}\right), v \leq 18$, configurations considered here has a point transitive group of automorphisms.

For an extensive note about the history of the Cremona-Richmond configuration see the last section.

\subsection{The Triangle-Free Configuration $\left(17_{3}\right)$}

The triangle-free configuration $\left(17_{3}\right)$ has lines:

$\begin{array}{ccccccccccccccccc}16 & 5 & 11 & 14 & 6 & 17 & 8 & 15 & 9 & 4 & 1 & 10 & 2 & 7 & 12 & 3 & 13 \\ 1 & 1 & 1 & 2 & 2 & 3 & 3 & 4 & 4 & 5 & 5 & 6 & 6 & 7 & 7 & 8 & 10 \\ 2 & 4 & 6 & 8 & 10 & 12 & 14 & 8 & 10 & 9 & 11 & 9 & 14 & 11 & 15 & 16 & 13 \\ 3 & 5 & 7 & 9 & 11 & 13 & 15 & 12 & 14 & 15 & 16 & 13 & 16 & 12 & 17 & 17 & 17\end{array}$

It is combinatorially self-polar by the correspondence $1 \leftrightarrow 1,2 \leftrightarrow 2,3 \leftrightarrow 3, \ldots$

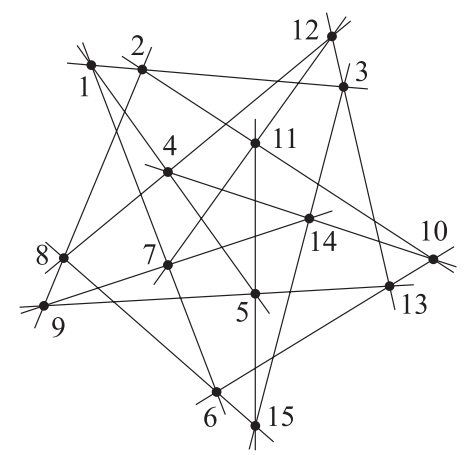

Fig. 2. A stellar realization of the Cremona-Richmond (153) configuration. 


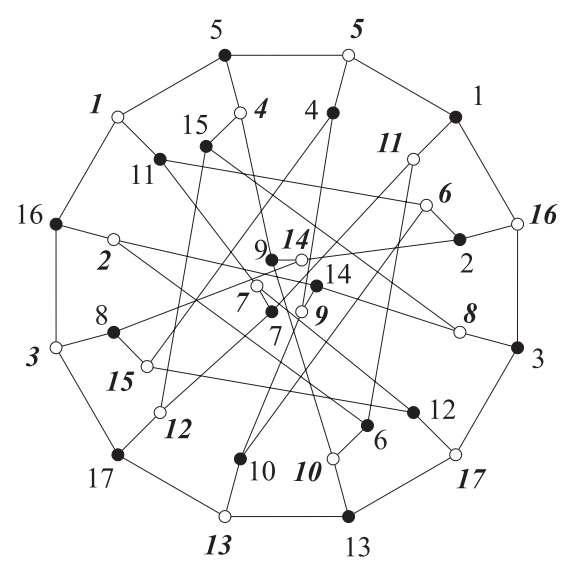

Fig. 3. Levi graph of the triangle-free combinatorially self-polar configuration (173).

Its group of automorphisms is the dihedral group $D_{6}$. The generators $a$ and $b$ satisfying the property $a^{2}=b^{2}=(a b)^{6}=e$ ( $e$ is the identity) are

$$
\begin{aligned}
& a=(2,4)(3,5)(8,10)(9,14)(11,12)(13,16), \\
& b=(1,3)(4,12)(5,13)(6,15)(7,14)(10,11)(16,17) .
\end{aligned}
$$

It has four point orbits, of three different sizes:

$$
\{1,3,13,17,16,5\},\{2,12,10,8,11,4\},\{7,14,9\},\{6,15\} .
$$

It also has four line orbits, with the same sets of lines. Its Levi graph is shown in Fig. 3, and a geometric realization is presented in Fig. 4.

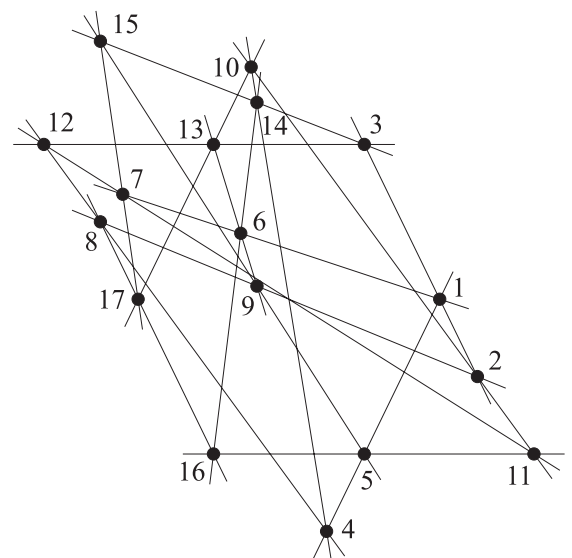

Fig. 4. A geometric realization of the triangle-free configuration (173). 


\subsection{The Triangle-Free Configuration 18-A}

The triangle-free configuration 18-A has lines

$\begin{array}{cccccccccccccccccc}5 & 1 & 4 & 9 & 13 & 11 & 16 & 6 & 7 & 2 & 3 & 8 & 12 & 10 & 14 & 17 & 18 & 15 \\ 1 & 1 & 1 & 2 & 2 & 3 & 3 & 4 & 4 & 5 & 5 & 6 & 6 & 7 & 7 & 8 & 10 & 12 \\ 2 & 4 & 6 & 8 & 10 & 12 & 14 & 8 & 10 & 9 & 11 & 9 & 11 & 13 & 14 & 15 & 17 & 16 \\ 3 & 5 & 7 & 9 & 11 & 13 & 15 & 12 & 14 & 13 & 16 & 17 & 15 & 18 & 16 & 18 & 18 & 17\end{array}$

It is combinatorially self-polar by the correspondence $1 \leftrightarrow 1,2 \leftrightarrow 2,3 \leftrightarrow 3, \ldots$ It has seven point orbits, of three different sizes:

$$
\{1,11,12,18\},\{2,8\},\{3,4,10,15\},\{5,6,13,17\},\{7,16\},\{9\},\{14\} .
$$

It also has seven line orbits, with the same sets of lines. Its automorphisms are the permutations: $e$ (identity), $a, b, c$, with $a^{2}=b^{2}=c^{2}=e, a b=b a=c$, which form the noncyclic group of four elements (dihedral $D_{2}$, Klein's "Vierergruppe"):

$$
\begin{aligned}
& a=(1,18)(2,8)(3,15)(4,10)(5,17)(6,13)(11,12), \\
& b=(1,11)(3,10)(4,15)(5,6)(7,16)(12,18)(13,17) .
\end{aligned}
$$

The Levi graph of the configuration 18-A is shown in Fig. 5 and its geometric realization in Fig. 6.

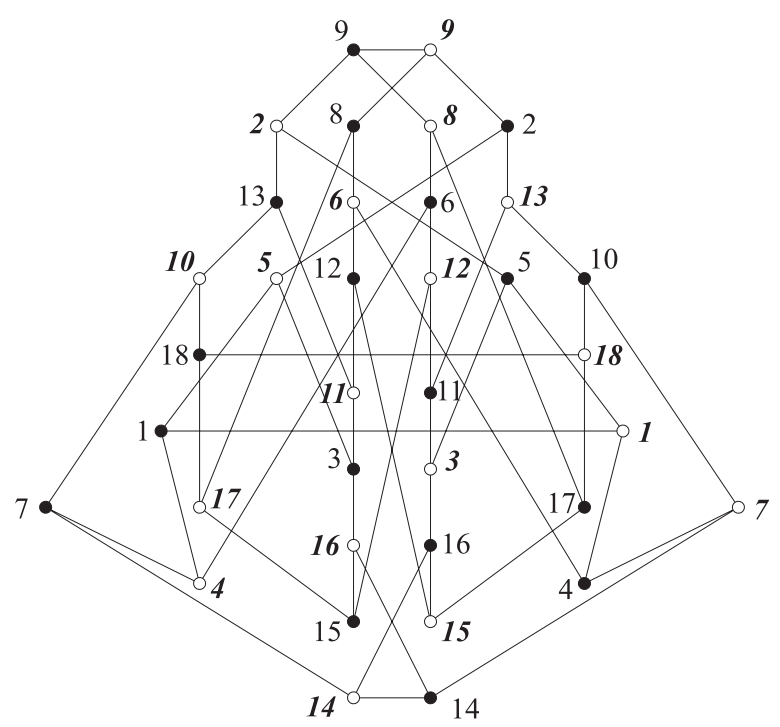

Fig. 5. The Levi graph of the configuration 18-A. 


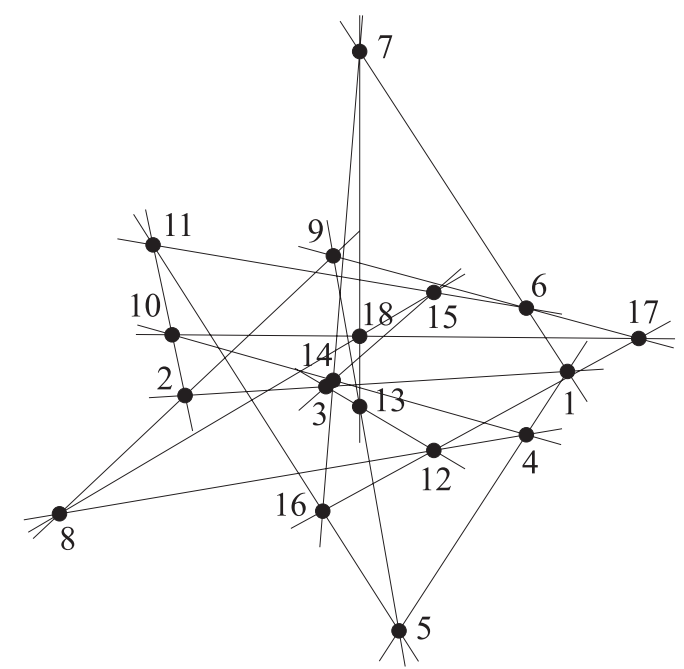

Fig. 6. A geometric realization of the 18-A configuration.

\subsection{The Triangle-Free Configuration 18-B}

The triangle-free configuration 18-B has lines:

$\begin{array}{cccccccccccccccccc}1 & 2 & 3 & 4 & 5 & 6 & 7 & 8 & 9 & 10 & 11 & 12 & 13 & 14 & 15 & 16 & 17 & 18 \\ 1 & 1 & 1 & 2 & 2 & 3 & 3 & 4 & 4 & 5 & 5 & 6 & 6 & 7 & 7 & 8 & 11 & 11 \\ 2 & 4 & 6 & 8 & 10 & 12 & 14 & 8 & 10 & 9 & 16 & 9 & 10 & 13 & 14 & 16 & 12 & 15 \\ 3 & 5 & 7 & 9 & 11 & 13 & 15 & 12 & 14 & 13 & 17 & 15 & 16 & 18 & 17 & 18 & 17 & 18\end{array}$

It has two point orbits:

$$
\{1,2,4,5,7,8,9,11,14,15,17,18\},\{3,6,10,12,13,16\},
$$

and three line orbits:

$$
\{1,3,5,7,8,9,10,11,12,14,16,17\},\{2,4,15,18\},\{6,13\}
$$

which shows that it is not self-dual. This is the smallest known configuration in which the number of line orbits is different from the number of point orbits. The automorphism group is $D_{12}$. The generators $a$ and $b$ satisfying $a^{2}=b^{2}=(a b)^{12}=e$ are

$$
\begin{aligned}
& a=(2,7)(3,6)(4,5)(8,17)(9,14)(10,13)(11,18)(12,16), \\
& b=(1,2)(4,9)(5,8)(6,10)(7,11)(12,13)(14,15)(17,18)
\end{aligned}
$$

The Levi graph of configuration 18-B is shown in Fig. 7 and its geometric realization in Fig. 8. It is of some interest that 18-B is a "double cover" of the Pappus configuration, 


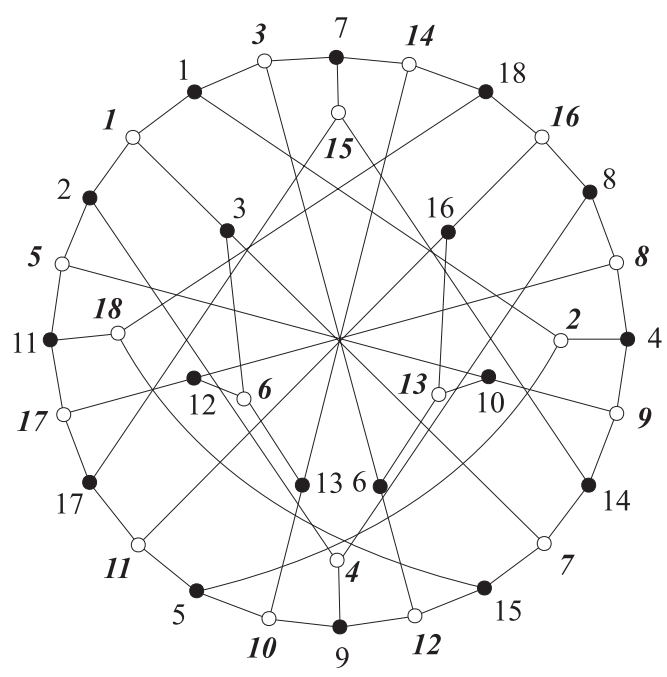

Fig. 7. The Levi graph of the configurations 18-B (upright) and 18-C (italic).

which results when the following identifications are performed:

$$
1=15,2=14,4=11,5=18,7=9,8=17 ; 3=16,6=12,10=13 .
$$

This "double cover" is shown in Fig. 9, where the identified points have been separated, and the lines have been replaced by pseudolines for better intelligibility. It should be pointed out that our "double cover" is not a covering projection in the usual sense of algebraic topology. Namely, it fails to be a local isomorphism at three singular points.

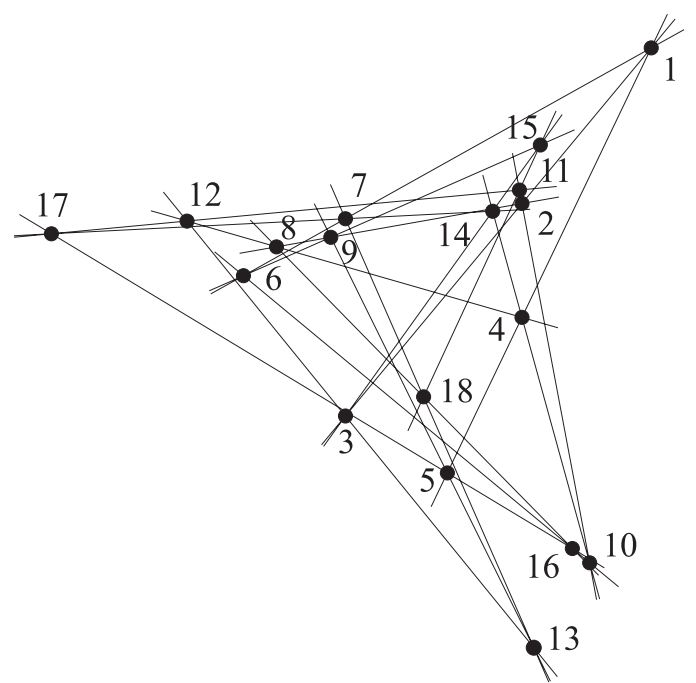

Fig. 8. A geometric realization of the 18-B configuration. 


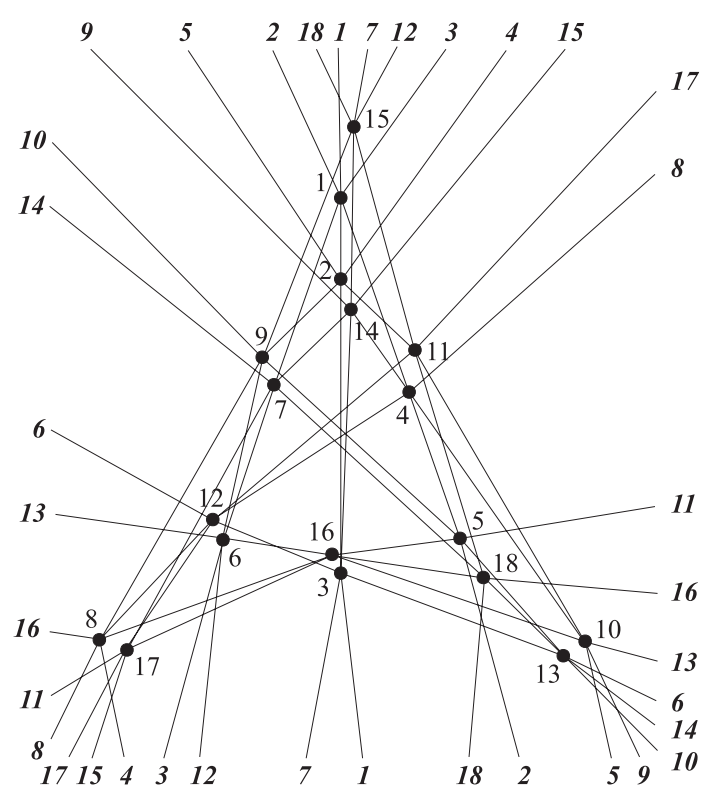

Fig. 9. A schematic diagram (pseudoline configuration) of the triangle-free 18-B configuration as a "double cover" of the Pappus configuration.

\subsection{The Triangle-Free Configuration $18-C$}

The triangle-free configuration $18-\mathrm{C}$ is dual to configuration 18-B. To underscore this, its points are denoted by italic, and its lines by upright characters. It has lines

$\begin{array}{cccccccccccccccccc}1 & 2 & 3 & 4 & 5 & 6 & 7 & 8 & 9 & 10 & 11 & 12 & 13 & 14 & 15 & 16 & 17 & 18 \\ 1 & 1 & 1 & 2 & 2 & 3 & 3 & 4 & 4 & 5 & 5 & 6 & 6 & 7 & 7 & 11 & 11 & 14 \\ 2 & 4 & 6 & 8 & 10 & 12 & 14 & 8 & 10 & 9 & 17 & 8 & 10 & 9 & 12 & 13 & 15 & 16 \\ 3 & 5 & 7 & 9 & 11 & 13 & 15 & 16 & 12 & 13 & 18 & 17 & 14 & 15 & 18 & 16 & 17 & 18\end{array}$

The configuration 18-C has three point orbits:

$$
\{1,3,5,7,8,9,10,11,12,14,16,17\},\{2,4,15,18\},\{6,13\}
$$

and two line orbits:

$$
\{1,2,4,5,7,8,9,11,14,15,17,18\},\{3,6,10,12,13,16\} .
$$

The Levi graph of configuration 18-C is shown in Fig. 7 and its geometric realization in Fig. 10. 


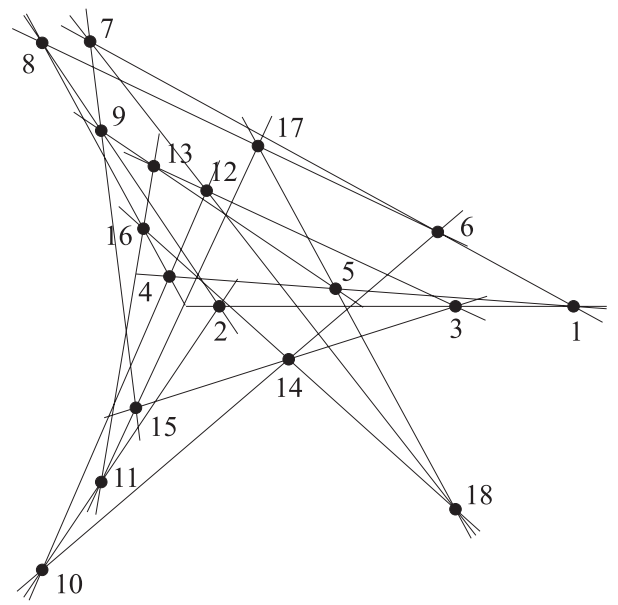

Fig. 10. A geometric realization of the $18-\mathrm{C}$ configuration.

\subsection{The Triangle-Free Configuration 18-D}

The triangle-free configuration 18-D has lines

$\begin{array}{cccccccccccccccccc}1 & 2 & 3 & 4 & 5 & 6 & 7 & 8 & 9 & 10 & 11 & 12 & 13 & 14 & 15 & 16 & 17 & 18 \\ 1 & 2 & 3 & 4 & 5 & 6 & 7 & 8 & 9 & 1 & 2 & 3 & 4 & 5 & 6 & 7 & 8 & 9 \\ 5 & 6 & 7 & 8 & 9 & 1 & 2 & 3 & 4 & 18 & 10 & 11 & 12 & 13 & 14 & 15 & 16 & 17 \\ 10 & 11 & 12 & 13 & 14 & 15 & 16 & 17 & 18 & 16 & 17 & 18 & 10 & 11 & 12 & 13 & 14 & 15\end{array}$

This configuration has two point orbits under its automorphisms, given by the permutations: $(1,2,3,4,5,6,7,8,9)$ and $(10,11,12,13,14,15,16,17,18)$, and the same orbits of lines. The automorphism group of order 18 given by the generators

$$
\begin{aligned}
& a=(2,9)(3,8)(4,7)(5,6)(10,15)(11,14)(12,13)(16,18), \\
& b=(1,2)(3,9)(4,8)(5,7)(10,16)(11,15)(12,14)(17,18)
\end{aligned}
$$

satisfying $a^{2}=b^{2}=(a b)^{9}=e$ is the dihedral group $D_{9}$.

The Levi graph of 18-D is shown in Fig. 11. It is in fact a generalized Petersen graph $G(18,5)$, which therefore is the smallest generalized Petersen graph that represents a triangle-free configuration $\left(v_{3}\right)$. A geometric realization of 18 -D as an astral configuration is given in Fig. 12. The symmetry group of this realization is the cyclic group $C_{9}$ of order 9 .

We may summarize the previous discussion in the form of a theorem.

Theorem 2. There exists a unique smallest astral triangle-free configuration $\left(18_{3}\right)$ and its Levi graph is the generalized Petersen graph $G(18,5)$.

Proof. Configuration 18-D satisfies the conditions of the theorem. Triangle-free configurations 18-A, 18-B, and 18-C lack the symmetries, while $\left(15_{3}\right)$ and $\left(17_{3}\right)$ configurations cannot be astral since 15 and 17 are odd. 


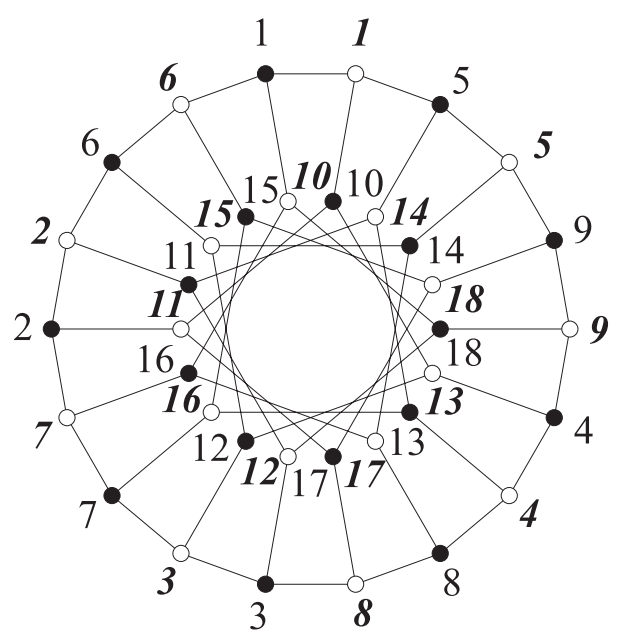

Fig. 11. The Levi graph of the triangle-free combinatorially self-polar configuration 18-D. This graph is the generalized Petersen graph $G(18,5)$.

\subsection{The Flag Transitive Triangle-Free Configuration $\left(20_{3}\right)$}

According to [1] there are altogether 14 triangle-free $\left(19_{3}\right)$ configurations and 162 triangle-free $\left(20_{3}\right)$ configurations. None of the triangle-free $\left(19_{3}\right)$ configurations is point or line transitive, but there is a unique triangle-free $\left(2_{3}\right)$ configuration which is flag transitive (no other triangle-free $\left(20_{3}\right)$ configuration is either point or line transitive). Its



Fig. 12. A geometrically self-polar astral realization of the triangle-free configuration $18-\mathrm{D}$. 
lines are

$\begin{array}{ccccccccccccccc}1 & 2 & 3 & 4 & 5 & 6 & 7 & 8 & 9 & 10 & 11 & 12 & 13 & 14 & 15 \\ 5 & 4 & 3 & 2 & 1 & 2 & 1 & 8 & 7 & 6 & 5 & 4 & 3 & 2 & 1 \\ 7 & 6 & 5 & 4 & 3 & 10 & 9 & 10 & 9 & 8 & 12 & 11 & 14 & 13 & 12 \\ 15 & 14 & 13 & 12 & 11 & 20 & 19 & 18 & 17 & 16 & 16 & 15 & 20 & 19 & 18\end{array}$

$$
\begin{array}{ccccc}
16 & 17 & 18 & 19 & 20 \\
10 & 9 & 8 & 7 & 6 \\
11 & 16 & 15 & 14 & 13 \\
17 & 20 & 19 & 18 & 17
\end{array}
$$

Its Levi graph is the Kronecker double cover of the dodecahedron graph $G(10,2)$, see Fig. 13. The configuration is combinatorially self-polar by the correspondence $1 \leftrightarrow 1$, $2 \leftrightarrow 2,3 \leftrightarrow 3, \ldots$ Its automorphism group of order 240 generated by

$$
\begin{aligned}
& a=(2,7)(4,17)(5,20)(9,12)(10,15)(13,14)(18,19), \\
& b=(1,2,3,4,5,6,7,8,9,10)(11,12,13,14,15,16,17,18,19,20)
\end{aligned}
$$

is $S_{5} \times C_{2}$.

Its (cyclic) astral realization is shown in Fig. 14. This configuration also admits a $d i$ hedral astral realization, i.e., an astral realization with a dihedral group of symmetries. In fact, there exists a one-parametric family of dihedral astral realizations. Two realizations from this family are shown in Fig. 15.

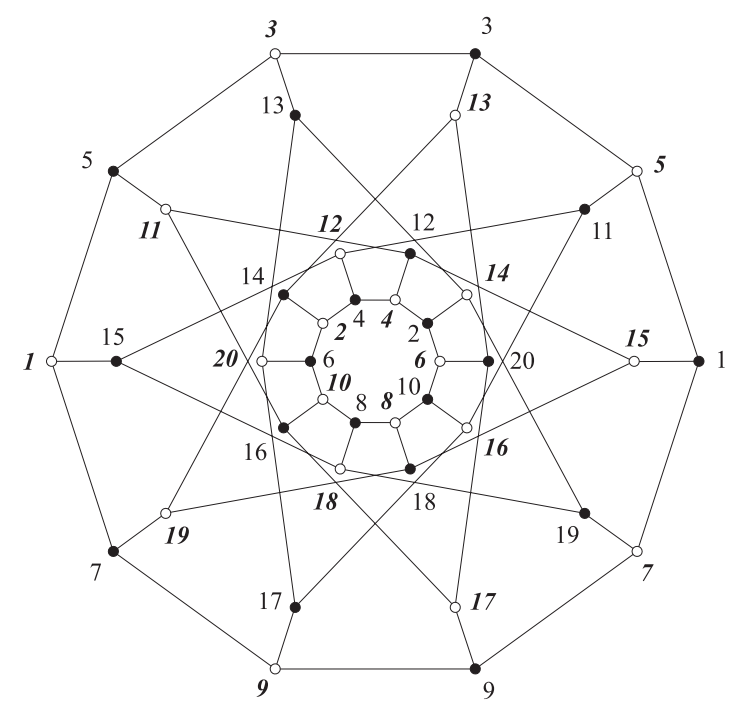

Fig. 13. The Levi graph of the flag transitive triangle-free cconfiguration $\left(20_{3}\right)$. It is the Kronecker double cover of the dodecahedron graph $G(10,2)$. 


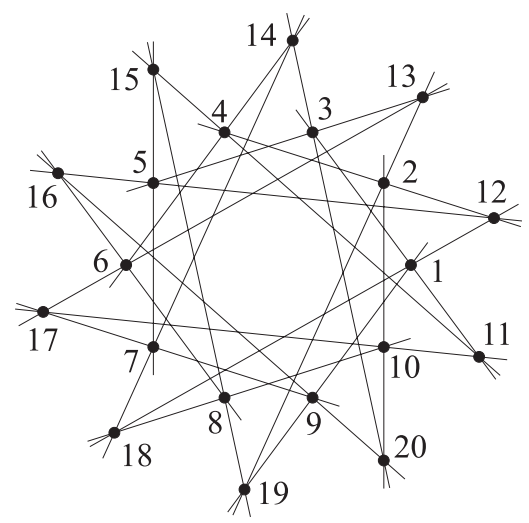

Fig. 14. A geometrically self-polar astral realization of the flag transitive triangle-free configuration $\left(20_{3}\right)$.

\subsection{The Point Transitive Triangle-Free Configuration $\left(21_{3}\right)$}

Among 4713 triangle-free $\left(21_{3}\right)$ configurations there exists a unique point transitive configuration, see [1]. Its lines are

$\begin{array}{ccccccccccccccc}1 & 2 & 3 & 4 & 5 & 6 & 7 & 8 & 9 & 10 & 11 & 12 & 13 & 14 & 15 \\ 1 & 1 & 4 & 6 & 8 & 2 & 12 & 7 & 15 & 11 & 5 & 14 & 9 & 3 & 2 \\ 2 & 4 & 6 & 8 & 10 & 10 & 13 & 13 & 16 & 16 & 18 & 19 & 20 & 17 & 6 \\ 3 & 5 & 7 & 9 & 11 & 12 & 14 & 15 & 17 & 18 & 19 & 20 & 21 & 21 & 18\end{array}$

$\begin{array}{cccccc}16 & 17 & 18 & 19 & 20 & 21 \\ 1 & 5 & 8 & 4 & 3 & 7 \\ 9 & 12 & 17 & 14 & 11 & 10 \\ 15 & 21 & 19 & 16 & 13 & 20\end{array}$
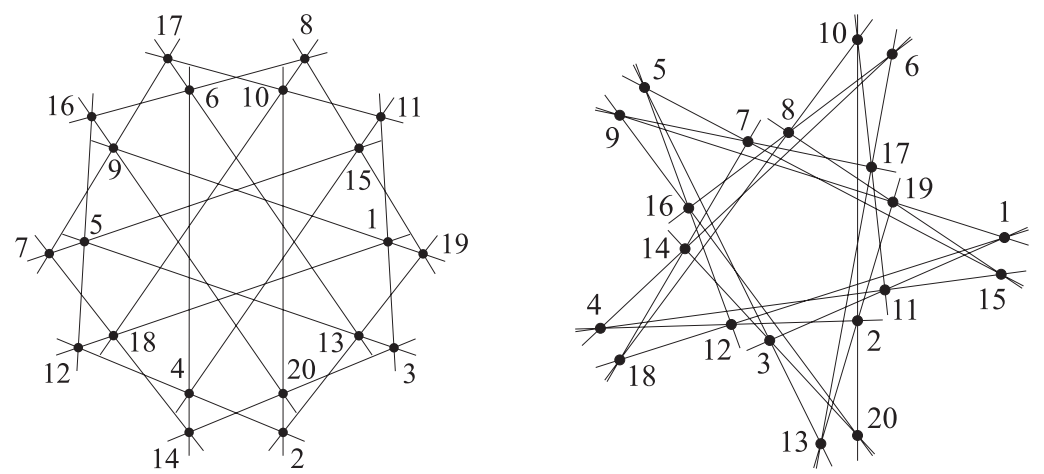

Fig. 15. Two dihedral astral realizations of the flag transitive triangle-free configuration $\left(20_{3}\right)$. 
Its Levi graph is shown in Fig. 16. While this configuration is point transitive, it is not line transitive; the two orbits are

$\{1,2,3,4,5,6,7,8,9,10,11,12,13,14\},\{15,16,17,18,19,20,21\}$.

Its automorphism group of order 42 is generated by

$$
\begin{aligned}
& a=(1,13,21,10,19,6,16)(2,14,9,11,5,7,17)(3,12,20,8,18,4,15), \\
& b=(1,2,3)(4,10,17)(5,12,21)(6,11,15)(7,8,16)(9,18,13)(14,20,19), \\
& c=(1,19)(2,14)(3,20)(4,18)(6,16)(7,11)(8,15)(9,17)(10,13) .
\end{aligned}
$$

It turns out that this group is the automorphism group of the dihedral group $D_{7}$.

Of course, it cannot be realized as an astral configuration, but it admits a stellar realization. It is shown in Fig. 17.

\section{Coordinates and Points}

It is well known that drawings of configurations can be misleading regarding the existence; this can happen either inadvertently (as in the well-known case of the ten configurations $\left(10_{3}\right.$ ) by Kantor [12]) or intentionally (as in [8]). Hence, the diagrams in Section 4 by themselves do not prove the existence of the geometric configurations in question. Therefore, to show their realizability, we give the coordinates of points for each of them. These values were obtained by the VEGA program using the algorithm described in [4].

Coordinates of points of the triangle-free $\left(17_{3}\right)$ configuration:

\begin{tabular}{cccccccccc}
\hline & \multicolumn{7}{c}{$i$} \\
\cline { 2 - 9 } & 1 & 2 & 3 & 4 & 5 & 6 & 7 & 8 & 9 \\
\hline$x_{i}$ & 1 & $\frac{5}{4}$ & $\frac{1}{2}$ & $\frac{1}{4}$ & $\frac{1}{2}$ & $-\frac{15}{47}$ & $-\frac{65}{59}$ & $-\frac{5}{4}$ & $-\frac{25}{118}$ \\
$y_{i}$ & 0 & $-\frac{433}{1000}$ & $\frac{433}{500}$ & $-\frac{1299}{1000}$ & $-\frac{433}{500}$ & $\frac{433}{1175}$ & $\frac{866}{1475}$ & $\frac{433}{1000}$ & $\frac{433}{5900}$ \\
\hline & & & & & $i$ & & & & \\
\cline { 2 - 10 } & 10 & 11 & 12 & 13 & 14 & 15 & 16 & 17 & \\
\hline$x_{i}$ & $-\frac{1}{4}$ & $\frac{13}{8}$ & $-\frac{13}{8}$ & $-\frac{1}{2}$ & $-\frac{25}{118}$ & $-\frac{5}{4}$ & $-\frac{1}{2}$ & -1 & \\
$y_{i}$ & $\frac{1299}{1000}$ & $-\frac{433}{500}$ & $\frac{433}{500}$ & $\frac{433}{500}$ & $\frac{1299}{1180}$ & $\frac{433}{300}$ & $-\frac{433}{500}$ & 0 & \\
\hline
\end{tabular}




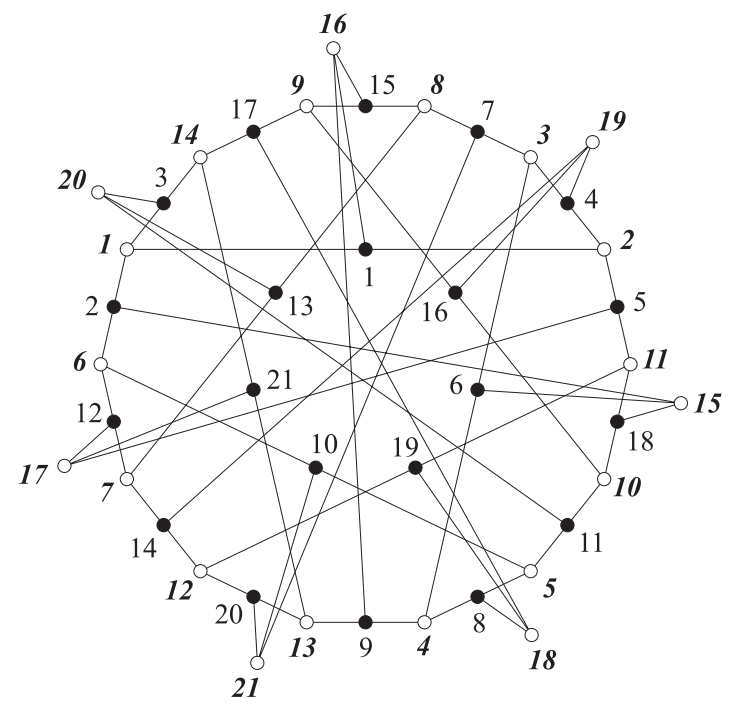

Fig. 16. The Levi graph of the unique point transitive triangle-free configuration $\left(21_{3}\right)$. The picture above is from [1] and was obtained by subdividing the Heawood graph (Levi graph of the Fano configuration) and connecting each set of three "collinear" vertices with valency 2 to a new vertex. The vertices of the Heawood graph correspond to the lines of the larger line orbit, the subdivision points correspond to the points, while the "new vertices" correspond to the lines of the smaller line orbit.

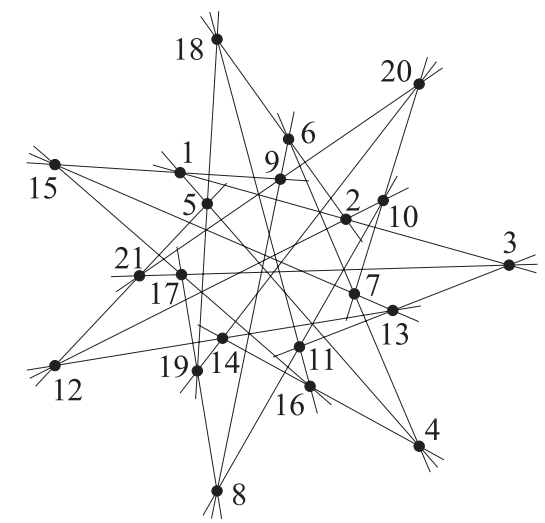

Fig. 17. A stellar realization of the unique point transitive triangle-free configuration $\left(21_{3}\right)$. 
Coordinates of points of the triangle-free configuration 18-A:

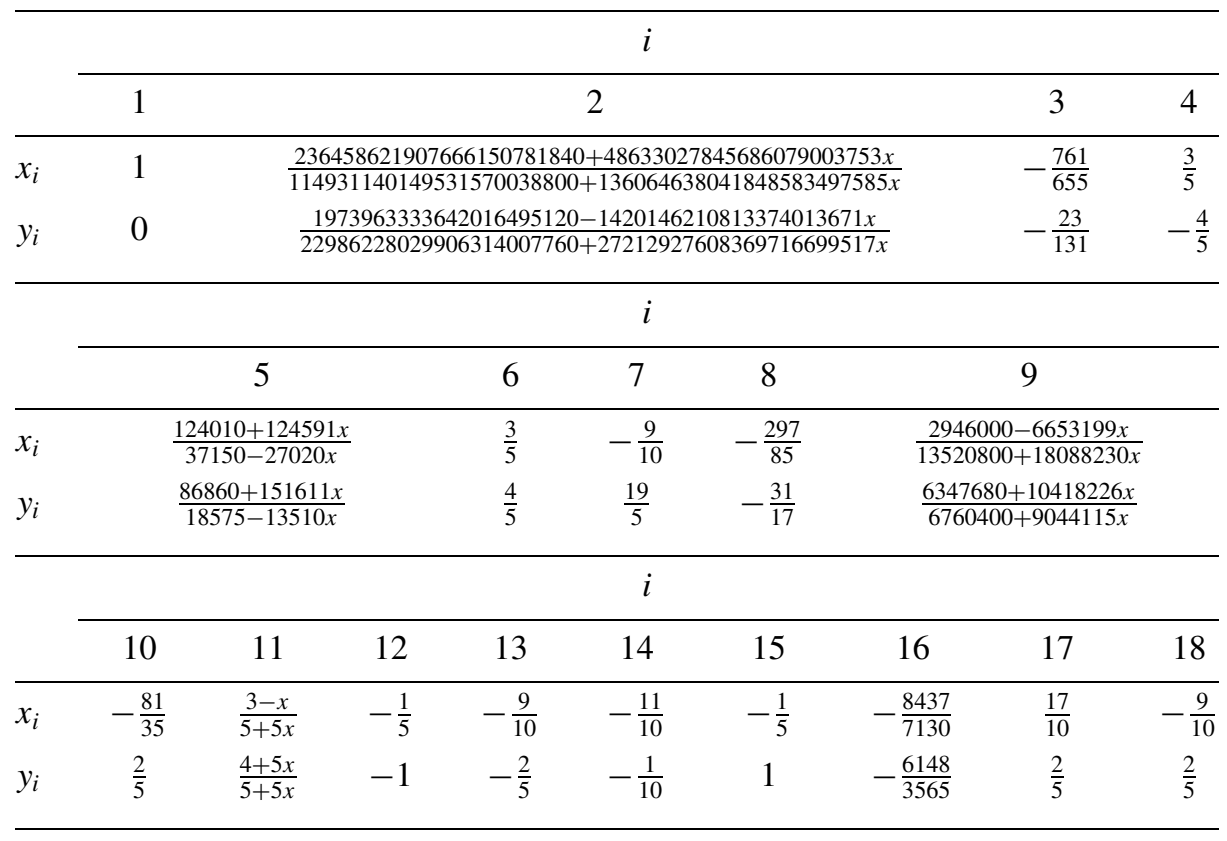

where $x$ is a root of the polynomial

$$
148356340695120+340383889522429 x+168711078656205 x^{2} .
$$

Note that this polynomial has two different roots, which give two different realizations. This is also true for the other two cases below.

Approximate coordinates of this realization are (taking $x=-1.38064$ ):

\begin{tabular}{|c|c|c|c|c|c|c|c|c|c|}
\hline & \multicolumn{9}{|c|}{$i$} \\
\hline & 1 & 2 & 3 & 4 & 5 & 6 & 7 & 8 & 9 \\
\hline$x_{i}$ & 1. & -2.3218 & -1.1618 & 0.6 & -0.6448 & 0.6 & -0.9 & -3.4941 & -1.0593 \\
\hline \multirow[t]{3}{*}{$y_{i}$} & 0. & -0.2698 & -0.1758 & -0.8 & -3.2895 & 0.8 & 3.8 & -1.8235 & 1.4034 \\
\hline & \multicolumn{9}{|c|}{$i$} \\
\hline & 9 & 10 & 12 & 13 & 14 & 15 & 16 & 17 & 18 \\
\hline$x_{i}$ & -2.3143 & -2.3017 & -0.2 & -0.9 & -1.1 & -0.2 & -1.1833 & 1.7 & -0.9 \\
\hline$y_{i}$ & 0.4 & 1.5254 & -1 & -0.4 & -0.1 & 1. & -1.7245 & 0.4 & 0.4 \\
\hline
\end{tabular}


Coordinates of vertices of the triangle-free configuration 18-B:

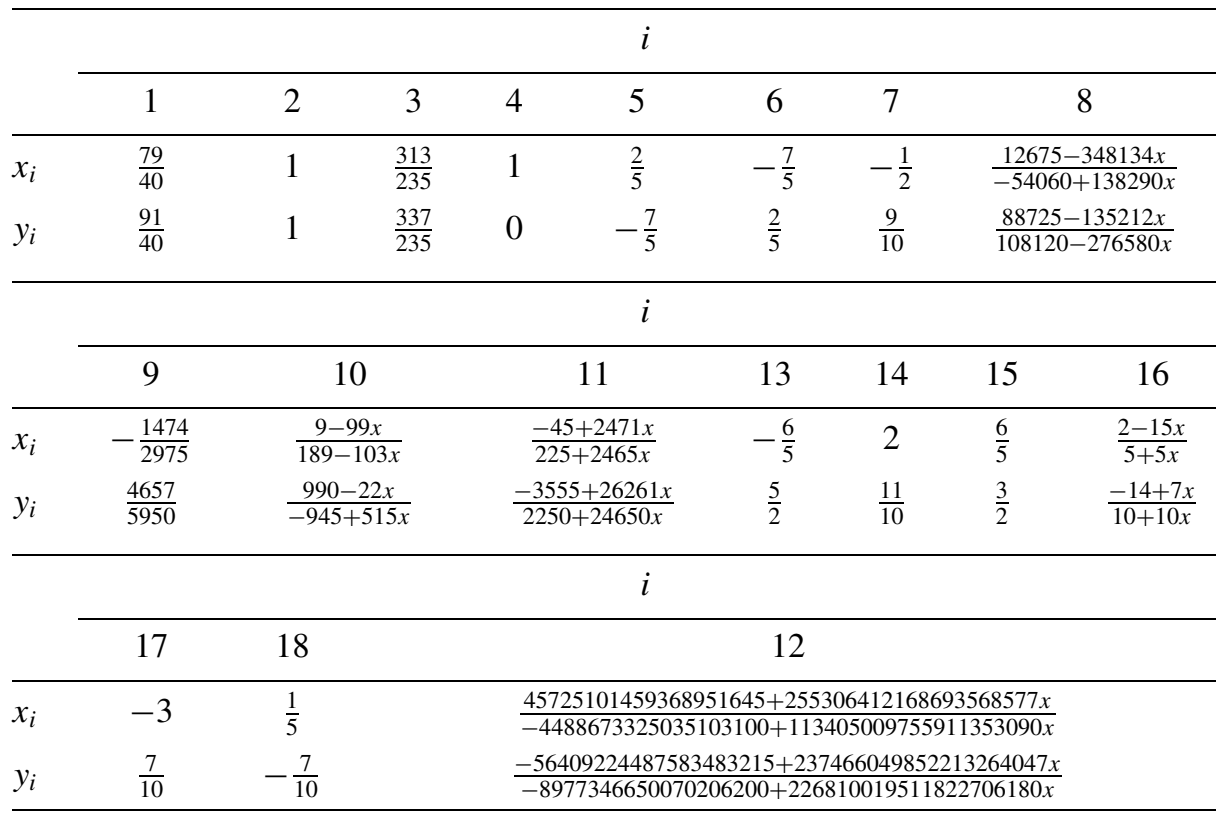

where $x$ is a root of the polynomial

$$
-13479623775-957906403010 x+2628530183883 x^{2} .
$$

Approximate values (taking $x=-0.0135669$ ) are:

\begin{tabular}{|c|c|c|c|c|c|c|c|c|c|}
\hline & & & & & $i$ & & & & \\
\hline & 1 & 2 & 3 & 4 & 5 & 6 & 7 & 8 & 9 \\
\hline$x_{i}$ & 1.975 & 1. & 1.3319 & 1. & 0.4 & -1.4 & -0.5 & -0.3110 & -0.4955 \\
\hline$y_{i}$ & 2.275 & 1. & 1.4340 & 0. & -1.4 & 0.4 & 0.9 & 0.8095 & 0.7827 \\
\hline & & & & & $i$ & & & & \\
\hline & 9 & 10 & 12 & 13 & 14 & 15 & 16 & 17 & 18 \\
\hline$x_{i}$ & 0.0543 & -0.4099 & -7.0118 & -1.2 & 2. & 1.2 & 0.4468 & -3 & 0.2 \\
\hline$y_{i}$ & -1.0402 & -2.0418 & 4.9468 & 2.5 & 1.1 & 1.5 & -1.4289 & 0.7 & -0.7 \\
\hline
\end{tabular}


Coordinates of points of the triangle-free configuration 18-C:

\begin{tabular}{|c|c|c|c|c|c|c|c|c|c|}
\hline & & & & & $i$ & & & & \\
\hline & 1 & 2 & 3 & 4 & 5 & & 6 & & \\
\hline$x_{i}$ & $\frac{3}{2}$ & 0 & $\frac{3}{4}$ & $-\frac{29}{170}$ & $\frac{1}{2}$ & & $\frac{758+7}{905+1}$ & & \\
\hline$y_{i}$ & 0 & 0 & 0 & $\frac{71}{425}$ & $\frac{1}{10}$ & & $\frac{71-5}{810+2}$ & & \\
\hline & & & & & $i$ & & & & \\
\hline & & & 7 & & & 8 & 9 & 10 & 11 \\
\hline$x_{i}$ & & $\frac{144803852}{65240188}$ & $4+3$ & $\frac{54635205}{6652218}$ & & $-\frac{4}{5}$ & $-\frac{2}{5}$ & $-\frac{4}{5}$ & $-\frac{2}{5}$ \\
\hline$y_{i}$ & & $\frac{84822016}{65240188}$ & $\frac{3+2}{4+1}$ & $\frac{65499569}{6652218}$ & & $\frac{13}{10}$ & $\frac{13}{20}$ & $-\frac{13}{10}$ & $-\frac{13}{20}$ \\
\hline & & & & & $i$ & & & & \\
\hline & 12 & 13 & & 14 & 15 & 16 & 17 & 18 & \\
\hline$x_{i}$ & $\frac{1065949}{21301980}$ & $-\frac{283}{1000}$ & & $\frac{+2332 x}{1+1320 x}$ & $\frac{-2+x}{5+5 x}$ & $-\frac{3}{10}$ & $\frac{1}{5}$ & $\frac{4}{5}$ & \\
\hline$y_{i}$ & $\frac{9544093}{21301980}$ & $\frac{1157}{2000}$ & & $\frac{-976 x}{0+660 x}$ & $\frac{-13+16 x}{20+20 x}$ & $\frac{2}{5}$ & $\frac{4}{5}$ & $-\frac{3}{5}$ & \\
\hline
\end{tabular}

where $x$ is a root of the polynomial

$$
-374743077758166+284285457716057 x+40064761902780 x^{2} .
$$

Approximate values (taking $x=-8.23189$ ) are:

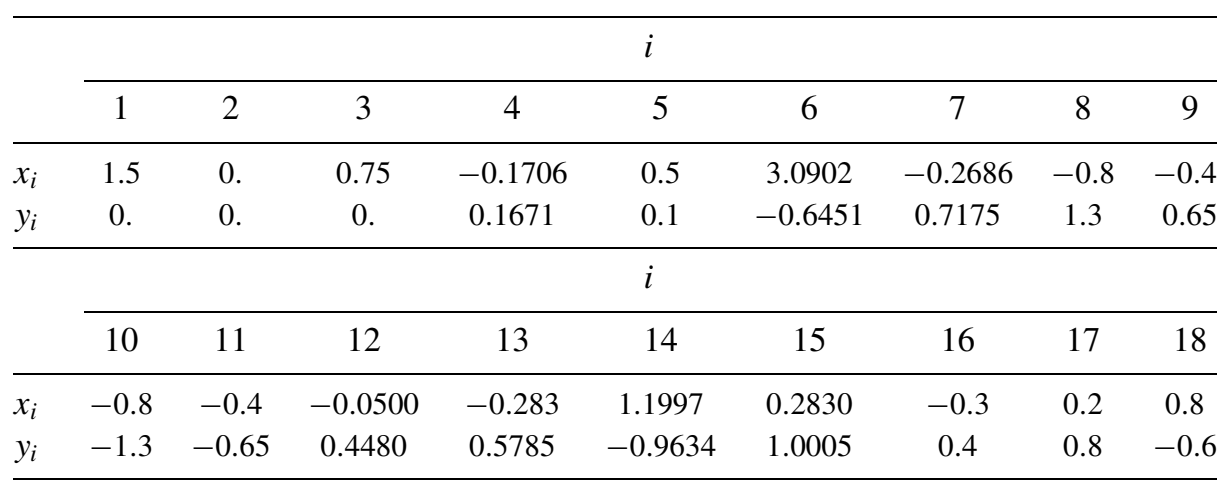

As for the configuration 18-D, a simpler description is possible due to its high degree of symmetry. The outer nine vertices are those of a regular nonagon, as are the inner nine. The lines passing through pairs of outer vertices are determined by the sides of a regular $\{9 / 4\}$-gon, those passing through two of the inner vertices by the sides of a regular $\{9 / 2\}$-gon. Each inner vertex is situated on one of the former lines and divides the side of the $\{9 / 4\}$-gon in ratio $1: 2.637 \ldots$ 
Analogously, for the configuration $\left(20_{3}\right)$ in Fig. 14 we have a regular $\{10 / 4\}$-gon surrounding a regular $\{10 / 2\}$-gon, such that the inner vertices divide the long sides in ratio $1: 3.57433 \ldots$ The construction of the configurations in Figs. 15 and 17 proceeds similarly, but requires the determination of two constants each.

\section{On the History of the Cremona-Richmond Configuration}

Unlike other configurations mentioned in the paper, the smallest triangle-free $\left(v_{3}\right)$ configuration, the Cremona-Richmond configuration, has a long history.

Although the name Cremona-Richmond configuration for the smallest triangle-free configuration is used by some writers, there seems to be no easily available explanation for the name. As we were curious, we investigated and here is what we found.

The beginnings of the history of this configuration are somewhat opaque. The first mentions of objects that appear related to the configuration are in the plethora of writings about cubic surfaces that were fashionable in the second half of the nineteenth century. It seems that the set of people who thoroughly understand these papers and books is today very small; it certainly does not include the authors of the present note. Another complication in tracing the history is that the papers in question appeared well before the concept of configurations was formulated by Reye [20] in 1876.

In connection with studies of the families of straight lines on cubic surfaces, it was noted by Schläfli [22, p. 117] in 1858 that there exist such surfaces on which it is possible to find families of 15 lines and 15 special tangent planes with the correct number of mutual incidences. A somewhat more accessible account of this is in [23], see in particular p. 197. Cremona [7] seems to have been the first to give an explicit list (on pp. 111-112) of the incidences of these lines and planes. From our perspective, with the concept of configuration available, this can be interpreted in two ways. On the one hand, from the lines and planes in question, by intersection with a suitable plane, one can obtain our configuration $\left(15_{3}\right)$ of points and lines. On the other hand, it is possible to interpret Cremona's list as a combinatorial configuration.

Coming from a somewhat different point of view, the Cremona-Richmond configuration was considered by Martinetti [16], who stressed that it is triangle-free. He also studied the triangle-free configurations $\left(n_{3}\right)$ for $16 \leq n \leq 18$; his enumeration is consistent with the one in [1]. However, although Martinetti's presentation is couched in geometric terminology (points, lines, polygons, etc.), his treatment is purely combinatorial. It is appropriate to recall that this was before the discovery by Schroeter [24] that one of the ten combinatorial configurations $\left(10_{3}\right)$ cannot be geometrically realized; hence Martinetti may possibly have thought that the establishment of a combinatorial configuration implies the existence of a configuration of real points and lines. We guess that his name is not attached to the configuration for two reasons. First, the paper [16] seems to have been largely forgotten; it is not mentioned in the survey [25], which is the basic reference for configurations. Second, as mentioned in the report [13] on [16], some of Martinetti's claims are incorrect.

In the following years, the next mention and description of the triangle-free geometric configuration $\left(15_{3}\right)$ as consisting of points and lines appears in the 1900 paper [21] by 
Richmond (his theorem on p. 128). This seems to have earned him the inclusion of his name in the designation of the configuration.

Visconti [27] in 1916 found that the configuration can be presented as a self-inscribed/ circumscribed polygon (with a cycle of vertices such as $(1,4,8,15,5,13,12,11,2,9,7$, $6,10,14,3$ ) in the notation of our Fig. 2), but does not admit a presentation as three pentagons inscribed cyclically into each other. She also discovered the astral character of the configuration we denoted $18-\mathrm{D}$, albeit only in the combinatorial version of the configuration.

None of these works contains any depiction of the Cremona-Richmond configuration. Zacharias [30] rediscovered the Cremona-Richmond configuration, and found a stellar realization for it; he seems not to have been aware of prior results concerning the configuration and, in particular, does not mention that it is triangle-free. Another geometric realization appears on p. 40 of [29]; it is not stellar, but Wells notes that it is triangle-free. The stellar realization of the configuration in Fig. 2 is from [3] and [18].

Some general questions arise naturally in connection with the consideration of the particular configurations. For example, is there a way of determining how symmetric can a geometric realization of a configuration be, given the knowledge of the automorphism groups, the point- and line-orbits, and whatever other information is inherent in the combinatorial description? In the specific cases considered here, can one find "nicer" realizations than the ones given in Figs. 4, 6, 8, and 10?

The triangle-free configurations $\left(v_{3}\right)$ considered here can be thought of as one extreme, to be contrasted with those configurations that have the maximal possible number of triangles for a given $v$. This question seems not to have been considered in the literature.

\section{Acknowledgement}

The first contacts leading to the present work were made during the geometry conference in Ein Gev in April 2000, organized by Prof. J. Zaks.

\section{References}

1. A. Betten, G. Brinkmann, and T. Pisanski, Counting symmetric configurations $v_{3}$, Discrete Appl. Math. 99 (2000), 331-338.

2. M. Boben, B. Grünbaum, and T. Pisanski, What did Steinitz prove in his thesis? (in preparation).

3. M. Boben and T. Pisanski, Polycyclic configurations, Eur. J. Combin. 24 (2003), 431-457.

4. J. Bokowski and B. Sturmfels, Computational Synthetic Geometry, Lecture Notes in Mathematics 1355, Springer, Heidelberg, 1989.

5. H. S. M. Coxeter, Self-dual configurations and regular graphs, Bull. Amer. Math. Soc. 56 (1950), 413-455.

6. H. S. M. Coxeter, The generalized Petersen graph G(24, 5), Comp. Math. Appl. 12B (1986), 579-583.

7. L. Cremona, Mémoire de géométrie pure sur les surfaces du troisieme ordre, J. Reine Angew. Math. 68 (1868), 1-133.

8. H. L. Dorwart and B. Grünbaum, Are these figures oxymora?, Math. Mag. 65 (1992), 158-169.

9. R. Frucht, J. E. Graver, and M. E. Watkins, The groups of the generalized Petersen graphs, Math. Proc. Cambridge Philos. Soc. 70 (1971), 211-218.

10. H. Gropp, On the existence and nonexistence of configurations $n_{k}$, J. Combin. Inform. System Sci. 15 (1990), 34-48. 
11. B. Grünbaum, Astral ( $\left.n_{k}\right)$ configrations, Geombinatorics 3 (1993), 32-37.

12. S. Kantor, Die Configurationen (3, 3)10, K. und k., Acad. Wiss. Vienna S.-ber. math-nat. Cl. 84 II (1881), 1291-1314.

13. E. Kötter, Review of [16], Jahrbuch Fortschritte Math. 18 (1886), 517.

14. F. W. Levi, Geometrische Konfigurationen, Hirzel, Leipzig, 1929.

15. F. W. Levi, Finite Geometrical Systems, University of Calcutta, Calcutta, 1942.

16. V. Martinetti, Sopra alcune configurazioni piane, Ann. Mat. pura Appl. (2) 14 (1886), 161-192.

17. T. Pisanski and coworkers, VEGA, a programming tool for manipulating discrete mathematical structures, see http://vega.ijp.si.

18. T. Pisanski, M. Boben, D. Marušič, A. Orbanić, and A. Graovac, The 10-cages and derived configurations, Discrete Math. 275 (2004), 265-276.

19. T. Pisanski, M. Boben, and A.Žitnik, Visualization of graphs and related discrete structures in Mathematica, Proc. PrimMath [2001] Conference, Zagreb 2001, pp. 27-39.

20. T. Reye, Geometrie der Lage I, 2nd edn., Rümpler, Hannover, 1876.

21. H. W. Richmond, On the figure of six points in space of four dimensions, Quart. J. Pure Appl. Math. 31 (1900), 125-160.

22. L. Schläfli, An attempt to determine the twenty-seven lines upon a surface of the third order, and to divide such surfaces into species in reference to the reality of the lines upon the surface, Quart. J. Pure Appl. Math. 2 (1858), 55-65, 110-120.

23. L. Schläfli, On the distribution of surfaces of the third order into species, in reference to the absence or presence of singular points, and the reality of their lines, Philos. Trans. Roy. Soc. London 153 (1863), 193-241.

24. H. Schroeter, Über lineare Konstructionen zur Herstellung der Konfigurationen $n_{3}$, Nachr. Ges. Wiss. Göttingen (1888), 237-253.

25. E. Steinitz, Konfigurationen der projektiven Geometrie, Encyclop. Math. Wiss. 3 (Geometrie) (1910), 481-516.

26. B. Sturmfels and N. White, All $11_{3}$ and $12_{3}$ configurations are rational, Aequationes Math. 39 (1990), 254-260.

27. E. Visconti, Sulle configurazioni piane atrigone, G. Mat. Battaglini 54 (1916), 27-41.

28. M. Watkins, A theorem on Tait coloring with an application to the generalized Petersen graphs, J. Combin. Theory 6 (1969), 152-164.

29. D. Wells, The Penguin Dictionary of Curious and Interesting Geometry, Penguin, London, 1991.

30. M. Zacharias, Streifzüge im Reich der Konfigurationen: Eine Reyesche Konfiguration (153), Stern- und Kettenkonfigurationen, Math. Nachr. 5 (1951), 329-345.

Received June 29, 2004, and in revised form September 7, 2005. Online publication January 20, 2006. 